\title{
The Iron Project and the RmaX Project
}

\author{
Anil K. Pradhan \\ Department of Astronomy, The Ohio State University, Columbus, $\mathrm{OH}$ \\ 43210, USA (www.astronomy.ohio-state.edu/ pradhan)
}

\begin{abstract}
Ongoing activities under an international collaboration of atomic physicists and astrophysicists under the Iron Project and the RmaX Project, with applications to X-ray astronomy, are briefly described.
\end{abstract}

\section{Introduction}

The Iron Project (IP; Hummer et al. 1993) is an extension of the erstwhile Opacity Project (OP; Seaton et al. 1994), devoted primarily to collisional and radiative processes of the Iron-peak elements. The $\mathrm{RmaX}$ Project is a part of the IP aimed at X-ray astronomy. The IP/RmaX work deals with highly charged ions and inner-shell processes.

To date 55 publications on Atomic Data From the Iron Project have appeared in Astronomy and Astrophysics. More details are on the IP website www.usm.uni-muenchen.de/people/ip/iron-project.html, or the author's website above. Additional details are provided in reviews in this volume by Palmeri and Mendoza on the OP/IP database TIPTOPBASE, and by Nahar on "New Radiative Data" not yet generally available. The IP/RmaX collaboration consists of about 20 members from Canada, France, Germany, UK, US, and Venezuela. Some RmaX publications are also reported in the Journal of Physics B: Atomic, Molecular, and Optical Physics.

\section{Methodology}

The IP/RmaX calculations, like the OP, are carried out using the R-matrix method, based on the close-coupling approximation from atomic collision theory (Burke and Robb 1975; Seaton 1987). Unlike the OP radiative calculations that were in LS coupling, the IP/Rmax calculations generally take account of fine structure and some relativistic effects using the Breit-Pauli R-matrix method (BPRM; Berrington et al. 1995).

\section{Radiative and Collisional Calculations}

One of the primary activities under the IP has been collisional calculations for all $\mathrm{Fe}$ ions (see references in the IP series), and radiative data for a many $\mathrm{Fe}$ and other ions.

Most recent collisional work has been on highly charged ions from $\mathrm{H}$-like to Ne-like sequences, and K- and L-shell radiative transitions. In the following 
subsections we exemplify the nature of the IP/RmaX work, on various atomic processes and using the same approximation (BPRM), for the important ion Ne-like Fe XVII that gives rise to a number of well known X-ray lines (see the Grotrian diagram in Chen et al. 2003).

\subsection{Electron impact excitation}

BPRM calculations for a benchmark study of electron scattering with Fe XVII showed extensive series of resonances that significantly enhance the effective (averaged) cross sections and rate coefficients (Fig. 1, Chen and Pradhan 2002; Chen et al. 2003). These calculations resolved longstanding discrepancies between two sets of experimental measurements using Electron-Beam-Ion-Traps (EBIT) at the Lawrence Livermore National Laboratory (Brown et al. 1998) and at the National Institute for Standards and Technology (NIST, Laming et al. 2000). The measured and calculated cross sections and line ratios in question are due to three prominent $\mathrm{x}$-ray transitions labeled $3 \mathrm{C}, 3 \mathrm{D}$, and $3 \mathrm{E}$, to the ground level $1 \mathrm{~s}^{2} 2 \mathrm{~s}^{2} 2 \mathrm{p}^{6}{ }^{1} \mathrm{~S}_{0}$ from excited levels:

$3 \mathrm{C}(\lambda 15.014 \AA) 1 \mathrm{~s}^{2} 2 \mathrm{~s}^{2} 2 \mathrm{p}^{5}[1 / 2] 3 \mathrm{~d}_{3 / 2}{ }^{1} \mathrm{P}_{1}^{o}, 3 \mathrm{D} \lambda 15.265 \AA 1 \mathrm{~s}^{2} 2 \mathrm{~s}^{2} 2 \mathrm{p}^{5}[3 / 2] 3 \mathrm{~d}_{5 / 2}{ }^{3} \mathrm{D}_{1}^{o}$, and $3 \mathrm{E} \lambda 15.456 \AA$ : $1 \mathrm{~s}^{2} 2 \mathrm{~s}^{2} 2 \mathrm{p}^{5}[3 / 2] 3 \mathrm{~d}_{5 / 2}{ }^{3} \mathrm{P}_{1}^{o}$. While the $3 \mathrm{C}$ is dipole allowed, the $3 \mathrm{D}$ and $3 \mathrm{E}$ are spin-forbidden inter-combination transitions. The so called ' $3 \mathrm{~s} / 3 \mathrm{~d}$ ' problem, also due to discrepancies between the two sets of EBIT measurements, has also been solved using (a) the Gaussian average, and (b) the Maxwellian average, over the cross sections in the collisional-radiative model (Chen and Pradhan, in preparation). Chen et al. (2003) discuss the factors that affect the accuracy of the collision strengths for many other transitions up to $n$ $=4$ levels in Fe XVII.

\subsection{Transition probabilities}

Relativistic BPRM transition probabilities for Fe XVII have been calculated for over $2.6 \times 10^{4}$ allowed (E1) transitions that are of dipole and inter-combination type, and about 3000 forbidden transitions that include electric quadrupole (E2), magnetic dipole (M1), electric octopole (E3), and magnetic quadrupole (M2) type, representing the most detailed calculations to date for the ion (Nahar et al. 2003).

\subsection{Photoionization and Electron-Ion Recombination}

BPRM photoionization and recombination calculations for (h $\nu+$ Fe XVII $\longleftrightarrow$ (e + Fe XVIII) have been reported by Zhang et al. (2001), using the unified method for $(e+$ ion $)$ recombination that includes both the radiative and the dielectronic recombination processes in an ab inito manner. Both the levelspecific and the total cross sections for the two inverse processes are obtained. In an earlier work, Pradhan et al. (2001) demonstrated that the theoretical unified rates agree with experimental data from ion storage rings to within $20 \%$. The unified and self-consistent approach to photoionization and $(\mathrm{e}+$ ion $)$ recombination is reviewed by Nahar and Pradhan (2003, astro-ph/0310624). 

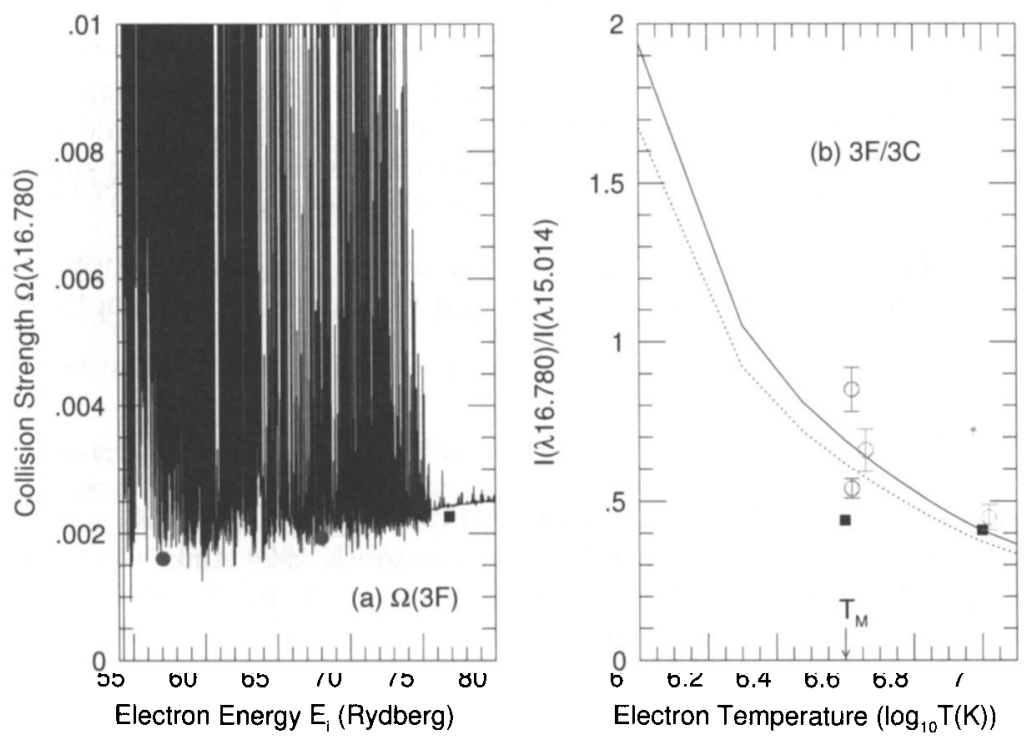

Figure 1. Enhancement of collisional rates of Fe XVII by resonances: (a) BPRM collision strength $\Omega$ for the forbidden $3 \mathrm{~F}$ line $2 s^{2} 2 p^{5} 3 s \rightarrow 2 s^{2} 2 p^{6}, J=$ $1 \rightarrow 0, \lambda 16.780 \AA$; the filled circles and square are non-resonant DW calculations; (b): line ratio $3 \mathrm{~F} / 3 \mathrm{C}$ vs. $\mathrm{T}$ from a 89 -level $\mathrm{C}-\mathrm{R}$ model. The electron densities for solid-line and dot-line curves are $10^{13}$ and $10^{9} \mathrm{~cm}^{-3}$ respectively. The 4 open circles with error bars are observed and experimental values: from the solar corona at $\mathrm{T}_{m} \sim 4 \mathrm{MK}$, from the corona of solar-type star Capella at $\sim 5 \mathrm{MK}$, and from the EBIT experiment at $0.9 \mathrm{keV}(\log \mathrm{T}=7)$. The filled squares are values using DW results. Owing to Maxwellian averaged rate coefficients, resonance enhancement is particularly large at low temperatures, such as in photoionized plasmas, as opposed to higher temperatures in coronal equilibrium. 


\subsection{Opacities, inner-shell excitation, and databases}

The review by Palmeri and Mendoza on TIPTOPBASE describes recent calculations on K-shell Auger processes and the Opacity Project/Iron Project atomic and opacities databases.

\section{Summary}

Atomic data for a variety of processes and ions are being calculated under the Iron/RmaX projects. The R-matrix approach is capable of taking account of all important atomic effects, and produce data of definitive accuracy that can be bencmharked against state-of-the-art experiments. Self-consistent ab initio calculations for Fe XVII are presented as an example of large-scale data obtained for all collisional and radiative processes in an ion using the same basic approximation (BPRM method) and wavefunction expansions.

Acknowledgments. The work reported herein is partially supported by the NASA Astrophysical Theory Program and the Space and Astrophysics Research program.

\section{References}

Berrington, K.A., Eissner, W.B., \& Norrington, P.H. 1995, CPC, 92, 290.

Brown G V, Beiersdorfer P, Liedahl D A, and Widmann K 1998 ApJ, 502, 1015

Burke, P.G. and Robb, W.D. 1975, Adv. At. Mol. Phys., 11, 143

Chen, G.X. and Pradhan, A.K. 2002 Phys.Rev.Lett, 89, 013202

Chen, G.X., Eissner, W., and Pradhan, A.K. 2003 J. Phys.B, 36, 453

Hummer D G, Berrington K A, Eissner W, Pradhan A K, Saraph H E, and Tully J A 1993, A\&A, 279, 298

Laming J M et al. $2000 \mathrm{ApJ}, 545$, L161

Nahar, S.N. and Pradhan, A.K. 2003, Self-consistent R-matrix Approach To Photoionization And Unified Electron-Ion Recombination, in "Radiation Processes in Physics and Chemistry", Elsevier (in press, Eds. R.H. Pratt and S. M. Manson), astro-ph/0310624.

Nahar, S.N., Chen, G.X., Eissner, W., Pradhan, A.K. 2003, A\&A, 408, 789 (astro$\mathrm{ph} / 0302561)$.

Pradhan, A.K., Nahar, S.N., and Zhang, H. L. 2001, ApJ, 549,L265

Seaton, M.J. 1987, J.Phys.B, 20, 6363

Seaton, M.J., Yu, Y., Mihalas, D. and Pradhan, A.K. 1994, MNRAS, 266, 805

Zhang, H.L., Nahar, S.N., Pradhan, A.K. 2001, Phys.Rev.A, 64, 032719. 